\title{
Prevalence of anti-hypertensive drug prescription in Thailand, a cross sectional study using National Health Security Office database
}

\author{
Theerut Luangmonkong ${ }^{1}$, Jiratchaya Wongsaardsakul1, Rujipas Laobutree ${ }^{1}$, Sarawut Jindarat ${ }^{2}$, Nattapon Jaisupa ${ }^{2 *}$ \\ 1 Department of Pharmacology, Faculty of Pharmacy, Mahidol University, Bangkok, Thailand \\ Department of Pharmacology, Phramongkutklao College of Medicine, Bangkok, Thailand
}

\begin{abstract}
Individual conditions are essential in pharmacotherapy of hypertension; however, information regarding the association between anti-hypertensive drugs and co-morbid diseases as recommended in clinical practice guidelines is not well acquainted. Therefore, this study aimed to report the prevalence of prescribed antihypertensive drugs using Thailand National Health Security Office database. In 2014, we found that calcium channel blockers (CCBs), angiotensin converting enzyme inhibitors (ACEIs), and diuretics, were prescribed in $56.11 \%, 42.70 \%$, and $33.58 \%$ of total patients, respectively. Similarly in 2015, CCBs and ACEIs were prescribed in $57.69 \%$ and $41.01 \%$ of total patients, whereas the $3^{\text {rd }}$ most frequently prescribed was anti-adrenergics $(28.55 \%)$. As recommended in the latest Thailand clinical practice guideline at that period, as a mono-therapy, patients younger than 55 years old without co-morbid diseases received either ACEIs (28.91\% and 28.40\%) or ARBs $(6.70 \%$ and $6.33 \%)$; however, CCBs were prescribed to these patients in the highest percentage $(33.25 \%$ and $40.35 \%$ in 2014 and 2015, respectively). Focusing on patients with co-morbid conditions, CCBs were prescribed at $54.23 \%$ and $55.72 \%$ in patients with diabetes mellitus and $62.53 \%$ and $62.58 \%$ in patients with chronic kidney diseases in 2014 and 2015, respectively. The percentages of patients with chronic kidney diseases who received CCBs were relatively higher than those treated with either ACEIs or ARBs, the first-line recommendation. Interestingly, we found the inappropriate combination between ACEIs and ARBs. Our finding revealed that although the anti-hypertensive drug prescription in Thailand was in line with the recommendations in clinical practice guidelines, certain problematic prescriptions remained available.
\end{abstract}

\section{Keywords:}

Anti-hypertensive drug, Thailand, National Health Security Office, Hypertension, Co-morbid diseases

\section{INTRODUCTION}

Hypertension is a chronic condition which the arterial blood pressure is consistently elevated. Although hypertension does not directly cause explicit symptoms, long-term high blood pressure is a major risk factor for cardiovascular diseases, cerebrovascular diseases, chronic kidney disease, and loss of vision ${ }^{1}$. According to the Thai guidelines on the treatment of hypertension by Thai Hypertension Society in 2019, hypertensive patients could have systolic blood pressure (SBP) or diastolic blood pressure (DBP) greater than or equal to 140 and $90 \mathrm{mmHg}$, respectively ${ }^{2}$. All of the diagnosed hypertensive patients should receive an appropriate management. Besides lifestyle modification, anti-hypertensive medicines play a major role in the treatment of hypertension ${ }^{1-2}$. Results from recent decades apparently demonstrated that the reduction of blood pressure by anti-hypertensive drugs was capable to reduce mortality from all causes ${ }^{3}$. There are 5 main classes of anti-hypertensive drugs; angiotensin converting enzyme inhibitors (ACEIs), angiotensin receptor blockers (ARBs), calcium-channel blockers (CCBs), beta-blockers, and diuretics. In addition, second line drugs that is alpha-blockers, vasodilators, central

\section{*Corresponding author:}

*Nattapon Jaisupa Email: nattapon.jai@pcm.ac.th, natta_diew@hotmail.com 
agonists, and peripheral adrenergic inhibitors (PAIs) were also used in a minor group of patients. To customize anti-hypertensive drugs, the average blood pressure, cardiovascular risk level, co-morbid diseases, and target organ damage are the pivotal factors for the selection of individual's best option ${ }^{1-2,4}$. In addition to patient's coexisting determinants, contraindications of each drug class are essential to be considered. To illustrate, ACEIs and $\mathrm{ARBs}$ which can retard kidney deterioration are the first agent to be recommended for the patients with diabetic mellitus $(\mathrm{DM})^{5}$. On the other hands, both ACEIs and ARBs are contraindicated in patients with bilateral renal artery stenosis and pregnancy. In uncontrolled patients, combining anti-hypertensive drugs is very common to synergize therapeutic efficacy. Nevertheless, certain drug combinations should be discouraged ${ }^{1-2}$. In this study, we aimed to analyze the prevalence of antihypertensive drugs prescription among Thai hypertensive patients with or without co-morbid diseases using Thailand National Health Security Office database in 2014 and 2015. Furthermore, the analytical results were discussed and compared with the clinical practice guidelines at that time.

\section{METHODS}

\subsection{Database and ethical approval}

Analyses were conducted using DMHTDAMUS (diabetes mellitus, hypertension-data archival for maximum utilization system), a database from Thailand National Health Security Office. This database was systematically collected and administered by Medical Research Network for Social Company Limited (MedResNet). The acquisition and processing of data in this research was approved by Institutional Review Board of Royal Thai Army Medical Department no. S056/61_Exp.

\subsection{Data sources and inclusion/exclusion criteria}

Database in DMHT-DAMUS was recruited from the medical record of hypertensive patients in 887 and 996 sites, including community, general, and regional hospitals which are under the Ministry of Public Health (MOPH) of Thailand, in 2014 and 2015, respectively. Hypertensive patients were randomly selected, and individual information was recorded in a standardized report prior to summarizing by MedResNet. Due to data collection of DMHT-DAMUS was yearly basis, the data was separately analyzed within the same year. Included patients had been treated in the hospital for at least 12 months after initial diagnosis. The hypertensive patients who were included in other clinical researches were excluded. It is essential to indicate that drug classes were the information to be recorded in the database; thus, names, dosages, and duration of drugs for the treatment of individual were unknown. In addition, the DMHT-DAMUS database recorded beta-blockers and alpha-blockers as the same set data, anti-adrenergics.

\subsection{Statistical analysis}

Data was analyzed by SPSS version 23.0. The percentage and prevalence were calculated using the number of interest data and corresponding overall population, as indicated at the end of each table. Any missing data was included in the total amount of patients for the calculation of prevalence. The prevalence ratio and chi-square were used to indicate statistical differences between two sets of prevalence. In addition, the association between two sets of data was calculated using Spearman correlation coefficient. A $p$-value less than 0.05 was considered significant.

\section{RESULTS}

\subsection{Demographic data}

Total number of hypertensive patients in the database was 32,749 in 2014 and 31,808 in 2015 (Table 1). More than $60 \%$ of the patients was female. The average patient's age was about 65 years old, and around $30 \%$ was within 55 to 64 years of age. Average systolic/ diastolic blood pressure (SBP/DBP), measured at the hospital visit, was $133.23 / 81.09$ and $134.21 / 81.41 \mathrm{mmHg}$ in 2014 and 2015, respectively. Only $21.69 \%$ and $22.89 \%$ of these hypertensive patients had no co-morbid diseases, and hyperlipidemia was the most common co-morbid disease among the patients $(62.13 \%$ and $63.35 \%$, in 2014 and 2015, respectively). It should be noted that metabolic syndrome was not recognized as a co-morbid disease in DMHT-DAMUS in 2014. Regarding the payment of medical treatment, the expenditure of more than $70 \%$ of the patients was covered by the universal coverage scheme.

\subsection{Total anti-hypertensive drug prescription}

The total number of anti-hypertensive drug prescriptions, including both single drug use and combinations (at least two classes of anti-hypertensive medicines), was analyzed (Table 2). In both 2014 and 2015, the first and the second most frequently prescribed medication were CCBs and ACEIs, respectively. The third rank in 2014 was diuretics (33.58\%), whereas antiadrenergics, including alpha- and beta-blockers, were prescribed at $28.55 \%$ in 2015 (28.17\% for diuretics). ARBs were prescribed at $14.81 \%$ and $17.35 \%$ in 2014 and 2015 , respectively, whereas, in total, less than $5 \%$ of the patients received vasodilators, central agonists, or PAIs. 
Table 1. Demographic data.

\begin{tabular}{|c|c|c|c|}
\hline & Data & $\begin{array}{c}\mathbf{2 0 1 4} \\
\text { number }(\% *)\end{array}$ & $\begin{array}{c}\mathbf{2 0 1 5} \\
\text { number }(\% *)\end{array}$ \\
\hline \multicolumn{2}{|c|}{ Total patients } & 32,749 & 31,808 \\
\hline \multirow[t]{3}{*}{ Sex } & Male & $12,218(37.31)$ & $12,187(38.31)$ \\
\hline & Female & $20,521(62.66)$ & $19,620(61.68)$ \\
\hline & Not specified & $10 \quad(0.03)$ & $1(<0.01)$ \\
\hline \multicolumn{2}{|c|}{ Average age (years old) } & 65.27 & 65.35 \\
\hline \multirow[t]{10}{*}{ Age range } & Less than 25 & $17(0.05)$ & $10(0.03)$ \\
\hline & $25-34$ & $169(0.52)$ & $176 \quad(0.55)$ \\
\hline & $35-44$ & $1,437 \quad(4.39)$ & $1,399 \quad(4.40)$ \\
\hline & $45-54$ & $5,719(17.46)$ & $5,499(17.29)$ \\
\hline & $55-64$ & $9,834(30.03)$ & $9,450(29.71)$ \\
\hline & $65-74$ & $8,917(27.23)$ & $8,840(27.79)$ \\
\hline & $75-84$ & $5,613(17.14)$ & $5,435(17.09)$ \\
\hline & $85-94$ & $953(2.91)$ & $965 \quad(3.03)$ \\
\hline & More than 95 & $29(0.09)$ & $32(0.10)$ \\
\hline & Not specified & $61 \quad(0.19)$ & $2(0.01)$ \\
\hline \multicolumn{2}{|c|}{ Average systolic blood pressure (SBP, $\mathbf{m m H g}$ ) } & 133.23 & 134.21 \\
\hline \multirow[t]{6}{*}{ SBP range } & Less than 120 & $5,468(16.70)$ & $4,694(14.76)$ \\
\hline & $120-129$ & $7,451(22.75)$ & $6,787(21.34)$ \\
\hline & $130-139$ & $10,035(30.64)$ & $10,047(31.59)$ \\
\hline & $140-159$ & $8,200(25.04)$ & $8,510(26.75)$ \\
\hline & $160-179$ & $1,375(4.20)$ & $1,490 \quad(4.68)$ \\
\hline & More than 180 & $220(0.67)$ & $280 \quad(0.88)$ \\
\hline \multicolumn{2}{|c|}{ Average diastolic blood pressure (DBP, $\mathrm{mmHg}$ ) } & 81.09 & 81.41 \\
\hline \multirow[t]{6}{*}{ DBP range } & Less than 80 & $19,176(58.55)$ & $17,994(56.57)$ \\
\hline & $80-84$ & $7,332(22.39)$ & $7,171(22.54)$ \\
\hline & $85-89$ & $2,397 \quad(7.32)$ & $2,472 \quad(7.77)$ \\
\hline & $90-99$ & $3,333(10.18)$ & $3,592(11.29)$ \\
\hline & $100-109$ & $427(1.30)$ & $480 \quad(1.51)$ \\
\hline & More than 110 & $84 \quad(0.26)$ & $99 \quad(0.31)$ \\
\hline \multicolumn{4}{|c|}{ Co-morbid diseases } \\
\hline \multicolumn{2}{|c|}{ None } & $7,104(21.69)$ & $7,280(22.89)$ \\
\hline \multicolumn{2}{|c|}{ With co-morbid diseases** } & $25,645(78.31)$ & $24,528(77.11)$ \\
\hline \multicolumn{2}{|c|}{ Diabetes mellitus } & $4,228(12.91)$ & $4,169(13.11)$ \\
\hline \multicolumn{2}{|c|}{ Chronic kidney disease } & $5,660(17.28)$ & $4,770(15.00)$ \\
\hline \multicolumn{2}{|c|}{ Left ventricular hypertrophy } & $166(0.51)$ & $106 \quad(0.33)$ \\
\hline \multicolumn{2}{|c|}{ Ischemic heart disease } & $1,446 \quad(4.42)$ & 1,234 \\
\hline \multicolumn{2}{|c|}{ Hyperlipidemia } & $20,347(62.13)$ & $20,150(63.35)$ \\
\hline \multicolumn{2}{|c|}{ Metabolic syndrome } & None $* * *$ & $18,379(57.78)$ \\
\hline \multicolumn{4}{|c|}{ Payment types for medical treatment } \\
\hline \multicolumn{2}{|c|}{ Universal coverage scheme } & $24,537(74.92)$ & $23,042(72.44)$ \\
\hline \multicolumn{2}{|c|}{ Government officer } & $6,318(19.29)$ & $6,643(20.88)$ \\
\hline \multicolumn{2}{|c|}{ Social security scheme } & $1,385 \quad(4.23)$ & $1,506 \quad(4.73)$ \\
\hline \multicolumn{2}{|c|}{ Private/state organization scheme } & $177 \quad(0.54)$ & $161 \quad(0.51)$ \\
\hline \multicolumn{2}{|c|}{ Cash } & $136 \quad(0.42)$ & $175 \quad(0.55)$ \\
\hline \multicolumn{2}{|c|}{ Not specified } & $196(0.60)$ & $281 \quad(0.88)$ \\
\hline
\end{tabular}

*Percentage is calculated from total patients. **Each patient might have multiple co-morbid diseases. *** Metabolic syndrome was not recognized as a co-morbid disease in 2014.

Table 2. Anti-hypertensive drug prescription in total patients (including both a single drug use and combinations).

\begin{tabular}{|c|c|c|c|}
\hline Anti-hypertensive drug classes & $\begin{array}{c}\mathbf{2 0 1 4} \\
\text { number }(\% *)\end{array}$ & $\begin{array}{r}\mathbf{2 0} \\
\text { numbe }\end{array}$ & $\begin{array}{l}15 \\
\operatorname{er}(\% *)\end{array}$ \\
\hline Calcium channel blockers (CCBs) & $18,374 \quad(56.11)$ & 18,351 & $(57.69)$ \\
\hline Angiotensin converting enzyme inhibitors (ACEIs) & $13,983 \quad(42.70)$ & 13,044 & $(41.01)$ \\
\hline Diuretics & $10,996 \quad(33.58)$ & 8,960 & $(28.17)$ \\
\hline Anti-adrenergics & $10,046 \quad(30.68)$ & 9,082 & $(28.55)$ \\
\hline Angiotensin II receptor blockers (ARBs) & $4,851 \quad(14.81)$ & 5,520 & $(17.35)$ \\
\hline Vasodilators & $1,029 \quad(3.14)$ & 1,088 & $(3.42)$ \\
\hline Central agonists & $276 \quad(0.84)$ & 225 & $(0.71)$ \\
\hline Peripheral adrenergic inhibitors (PAIs) & $36 \quad(0.11)$ & 38 & $(0.12)$ \\
\hline Total patients & $32,749(100.00)$ & \multicolumn{2}{|c|}{$31,808(100.00)$} \\
\hline
\end{tabular}

*Percentage is calculated from total patients. Each patient might receive multiple drug classes. 
Table 3. Anti-hypertensive drug mono-therapy in patients without co-morbid diseases.

\begin{tabular}{|c|c|c|}
\hline Anti-hypertensive drug classes & $\begin{array}{c}\mathbf{2 0 1 4} \\
\text { number }(\% *)\end{array}$ & $\begin{array}{c}\mathbf{2 0 1 5} \\
\text { number }(\% *)\end{array}$ \\
\hline Calcium channel blockers (CCBs) & $1,195(16.82)$ & $1,540 \quad(21.15)$ \\
\hline Angiotensin converting enzyme inhibitors (ACEIs) & $818(11.51)$ & $892(12.25)$ \\
\hline Diuretics & $513 \quad(7.22)$ & $485 \quad(6.66)$ \\
\hline Anti-adrenergics & $289 \quad(4.07)$ & $303 \quad(4.16)$ \\
\hline Angiotensin II receptor blockers (ARBs) & $211 \quad(2.97)$ & $(3.50)$ \\
\hline Vasodilators & $13(0.18)$ & $(0.14)$ \\
\hline Central agonists & $1 \quad(0.01)$ & $(0.03)$ \\
\hline Peripheral adrenergic inhibitors (PAIs) & $0 \quad(0.00)$ & $0 \quad(0.00)$ \\
\hline Total patients without co-morbid diseases who received mono-therapy & $3,040 \quad(42.79)$ & $3,487 \quad(47.88)$ \\
\hline Total patients without co-morbid diseases & $7,104(100.00)$ & $7,280(100.00)$ \\
\hline
\end{tabular}

*Percentage is calculated from total patients without co-morbid diseases.

Table 4. Anti-hypertensive drug dual-therapy in patients without co-morbid diseases.

\begin{tabular}{|c|c|c|}
\hline Dual anti-hypertensive drug regimens & $\begin{array}{c}\mathbf{2 0 1 4} \\
\text { number }(\% *)\end{array}$ & $\begin{array}{c}\mathbf{2 0 1 5} \\
\text { number }(\% *)\end{array}$ \\
\hline CCBs+ACEIs & $831(11.70)$ & $918 \quad(12.61)$ \\
\hline CCBs+Diuretics & $463 \quad(6.52)$ & $376 \quad(5.16)$ \\
\hline CCBs+Anti-adrenergics & 333 (4.69) & $374 \quad(5.14)$ \\
\hline $\mathrm{CCBs}+\mathrm{ARBs}$ & $235 \quad(3.31)$ & $276 \quad(3.79)$ \\
\hline ACEIs+Diuretics & $465 \quad(6.55)$ & $356 \quad(4.89)$ \\
\hline ACEIs+Anti-adrenergics & $223(3.14)$ & $222 \quad(3.05)$ \\
\hline ACEIs+ARBs & $16 \quad(0.23)$ & $13(0.18)$ \\
\hline Diuretics+Anti-adrenergics & $269 \quad(3.79)$ & $186 \quad(2.55)$ \\
\hline Diuretics+ARBs & $102 \quad(1.44)$ & $79 \quad(1.09)$ \\
\hline Anti-adrenergic+ARBs & $38 \quad(0.96)$ & $85 \quad(1.17)$ \\
\hline Total patients without co-morbid diseases who received dual-therapy & $3,005 \quad(42.30)$ & $2,883 \quad(39.63)$ \\
\hline Total patients without co-morbid diseases & $7,104(100.00)$ & $7,280(100.00)$ \\
\hline
\end{tabular}

CCBs, calcium channel blockers; ACEIs, angiotensin converting enzyme inhibitors; ARBs, angiotensin II receptor blockers. *Percentage is calculated from total patients without co-morbid diseases.

Table 5. Anti-hypertensive drug mono-therapy in patients without co-morbid diseases dividend by age.

\begin{tabular}{|c|c|c|c|c|c|c|c|c|c|}
\hline \multirow[t]{2}{*}{$\begin{array}{l}\text { Anti-hypertensive } \\
\text { drug classes }\end{array}$} & \multicolumn{4}{|c|}{$\begin{array}{c}\mathbf{2 0 1 4} \\
\text { number }(\% *)\end{array}$} & \multicolumn{5}{|c|}{$\begin{array}{c}\mathbf{2 0 1 5} \\
\text { number }(\% *)\end{array}$} \\
\hline & Age $<55$ & \multicolumn{2}{|c|}{ Age $\geq 55$} & \multirow{2}{*}{$\begin{array}{c}\begin{array}{c}\text { Prevalence ratio } \\
(p \text {-value })\end{array} \\
0.86\left(<0.001^{\#}\right)\end{array}$} & \multicolumn{2}{|c|}{ Age $<55$} & \multicolumn{2}{|c|}{ Age $\geq 55$} & $\begin{array}{c}\text { Prevalence ratio } \\
(p \text {-value })\end{array}$ \\
\hline $\mathrm{CCBs}$ & $300 \quad(33.25)$ & 895 & $(40.89)$ & & 395 & $(40.35)$ & 1,145 & $(45.65)$ & $0.88 \quad\left(0.002^{\#}\right)$ \\
\hline ACEIs & $246 \quad(28.91)$ & 572 & (26.13) & $1.11(0.814)$ & 278 & $(28.40)$ & 614 & (24.48) & $1.16 \quad(0.109)$ \\
\hline Diuretics & $151 \quad(17.74)$ & 362 & $(16.54)$ & $1.07 \quad(0.883)$ & 123 & (12.56) & 362 & $(14.43)$ & $0.87 \quad(0.076)$ \\
\hline Anti-adrenergics & $95 \quad(11.16)$ & 194 & $(8.86)$ & $1.26(0.231)$ & 118 & (12.05) & 185 & $(7.38)$ & $1.63\left(<0.001^{\#}\right)$ \\
\hline ARBs & $(6.70)$ & 154 & (7.04) & $0.95 \quad(0.383)$ & 62 & $(6.33)$ & 193 & $(7.70)$ & $0.82 \quad(0.101)$ \\
\hline Vasodilators & $(0.24)$ & 11 & $(0.50)$ & $0.47 \quad(0.258)$ & 2 & $(0.20)$ & 8 & $(0.32)$ & $0.64 \quad(0.535)$ \\
\hline Central agonists & $\begin{array}{ll}0 & (0.00)\end{array}$ & 1 & $(0.05)$ & $0.00 \quad(0.515)$ & 1 & $(0.10)$ & 1 & $(0.04)$ & $2.56 \quad(0.510)$ \\
\hline Total patients & $851(100.00)$ & 2,189 & $(\mathbf{1 0 0 . 0 0 )}$ & 3,040 & 979 & $(100.00)$ & 2,508 & $(100.00)$ & 3,487 \\
\hline
\end{tabular}

CCBs, calcium channel blockers; ACEIs, angiotensin converting enzyme inhibitors; ARBs, angiotensin II receptor blockers. *Percentage is calculated from total patients in the same age group. ${ }^{*} p<0.05$ when compared the prevalence of patients age $<55$ to patients age $\geq 55$.

\subsection{Anti-hypertensive drug prescription in patients without co-morbid diseases}

We analyzed anti-hypertensive drug prescription in 7,104 and 7,280 patients who were diagnosed as hypertension without co-morbid diseases in 2014 and 2015, respectively (Table 3). These patients would receive one or more classes of anti-hypertensive drug irrespective of concurrent conditions. The number of patients received a single anti-hypertensive medicine (mono-therapy) in 2014 and 2015 were 3,040 (42.79\% of the patients without co-morbid diseases) and 3,487 (47.88\%), respectively. Again, CCBs were prescribed at the highest percentage among the patients received mono-therapy (16.28\% in 2014 and $21.15 \%$ in 2015). The next was ACEIs (11.51\% and $12.25 \%)$, diuretics (7.22\% and 6.66\%), anti-adrenergics (4.07\% and $4.16 \%$ ), and ARBs (2.97\% and 3.50\%) in 2014 and 2015, respectively. As a mono-therapy, vasodilators $(0.18 \%$ and $0.14 \%)$ and central agonists $(0.01 \%$ and $0.03 \%)$ were scarcely prescribed in 2014 and 2015, respectively, while PAIs were not given. 
Next, we considered the number of dualtherapy prescription in patients without co-morbid diseases (Table 4) and found that 3,005 (42.30\% of all patients without co-morbid diseases in 2014) and 2,883 (39.63\% in 2015) patients received regimens comprising of either CCBs, ACEIs, diuretics, anti-adrenergics, or ARBs. The highest number of dual-therapy prescription was CCBs plus ACEIs in both 2014 (11.70\%) and 2015 $(12.61 \%)$. It should be note that the number of patients received other combinations including vasodilators, central agonists, and PAIs was not shown due to the amount was negligible.

In addition, we analyzed the triple-therapy prescription in patients without co-morbid diseases. Due to a plenty of treatment regimens were seen, hereby, we merely reported two most common prescribed regimens with diuretics, the recommended third drug class in the latest Thailand clinical practice guidelines at that time ${ }^{1-}$ 2,6 . In 2014, the combination of CCBs, ACEIs, and diuretics was prescribed in 228 patients (3.21\% of total patients without co-morbid diseases), and the combination of CCBs, ARBs, and diuretics was prescribed in 51 patients $(0.72 \%)$. Similarly in 2015 , the first combination was prescribed in 186 patients $(2.55 \%)$, and the latter was prescribed in 45 patients $(0.62 \%)$.

Due to age $(<55$ years and $\geq 55$ years) was suggested as a criterion for the selection of anti-hypertensive drug for individual without co-morbid diseases at that time ${ }^{6}$, we compared the prevalence of drug prescription in patients who received mono-therapy in two age groups (Table 5). The prevalence of CCBs in patients older than 55 years old was significantly higher than those of younger in both years.

\subsection{Anti-hypertensive drug prescription in patients with co-morbid diseases}

Hypertensive patients with DM, CKD, and left ventricular hypertrophy $(\mathrm{LVH})$ are recommended to receive the specific class of anti-hypertensive medicines which are potentially benefit in individual conditions ${ }^{1-}$
2,6. After DM was designated as the co-morbid disease, we categorized the prevalence of drug prescription in hypertensive patients (Table 6 ) and found that $54.23 \%$ and $55.72 \%$ of DM patients received CCBs in 2014 and 2015 , respectively. However, the total percentage of patients received either ACEIs or ARBs was higher than that received CCBs in both years. It should be noted that the percentages of patients with DM treated with ACEIs, ARBs, vasodilators, and central agonists were significantly higher than those of patients without DM. On the other hand, CCBs were prescribed in non-DM patients with a relatively higher percentage.

Next, the prevalence of drug prescription in patients with and without CKD was analyzed (Table 7). Again, $62.53 \%$ and $62.58 \%$ of CKD patients in 2014 and 2015, respectively, received CCBs. Although the percentages of patients received either ACEIs or ARBs were combined, the total percentage remained lower than those of CCBs. Also, we found that the hypertensive patients with CKD received CCBs, anti-adrenergics, vasodilators, and central agonists with a higher percentage than those without CKD. On the other hand, the proportion of CKD patients who received ACEIs was significantly lower than that of non-CKD patients in both years.

Since anti-hypertensive medicines could be simultaneously used for the management of LVH, this condition was another co-morbid disease of interest in our study (Table 8). Nevertheless, only 166 and 106 patients with LVH were found in 2014 and 2015, respectively, and we did not observe a clear difference on the prevalence of anti-hypertensive drug prescription.

\subsection{Correlation analysis with anti-hypertensive drug prescription}

Finally, we used the correlation analysis to elucidate whether the number of anti-hypertensive drugs that a patient received associated with either age, waist circumference, SBP, or DBP (Table 9). Although all analyses indicated positive correlation with statistically

Table 6. Anti-hypertensive drug prescription in patients with and without diabetes mellitus (DM).

\begin{tabular}{|c|c|c|c|c|c|c|c|c|c|c|}
\hline \multirow[t]{2}{*}{$\begin{array}{l}\text { Anti-hypertensive } \\
\text { drug classes }\end{array}$} & \multicolumn{5}{|c|}{$\begin{array}{c}\mathbf{2 0 1 4} \\
\text { number }(\% *)\end{array}$} & \multicolumn{5}{|c|}{$\begin{array}{c}\mathbf{2 0 1 5} \\
\text { number }(\% *)\end{array}$} \\
\hline & \multicolumn{2}{|c|}{ With DM } & \multicolumn{2}{|c|}{ Without DM } & \multirow[t]{2}{*}{$\begin{array}{c}\text { Prevalence ratio } \\
(p \text {-value })\end{array}$} & \multicolumn{2}{|c|}{ With DM } & \multicolumn{2}{|c|}{ Without DM } & $\begin{array}{c}\text { Prevalence ratio } \\
(p \text {-value })\end{array}$ \\
\hline $\mathrm{CCBs}$ & 2,293 & $(54$ & 16, & $(56$ & & 2,323 & $(55$. & 16,028 & (57.99) & $0.96 \quad\left(0.006^{\#}\right)$ \\
\hline ACEIs & 2,087 & (49. & & & $1.18(<0.0$ & 1,786 & & 11,258 & (40.73) & $1.05\left(0.010^{\#}\right)$ \\
\hline Diur & 1,343 & $(31.76)$ & 9,653 & (33. & $0.94 \quad(0.00$ & 1,153 & ) & 7,807 & $(28.25)$ & $0.98 \quad(0.430)$ \\
\hline Anti-ad & 1,313 & $(31.05)$ & 8,733 & (30.62) & $1.01 \quad 0.5$ & 1,277 & (30.63) & 7,805 & (28.24) & $1.08\left(0.001^{\#}\right)$ \\
\hline ARBs & 813 & (19.23) & 4,038 & (14.16) & $1.36\left(<0.001^{\#}\right)$ & 1,057 & $(25$. & 4,463 & (16.15) & $1.57\left(<0.001^{\#}\right)$ \\
\hline Vasodi & 209 & $(2.88)$ & 820 & $(4.94)$ & $1.72\left(<0.001^{\#}\right)$ & 212 & $(5.09)$ & 876 & $(3.17)$ & $1.61\left(<0.001^{\#}\right)$ \\
\hline Central agonists & 48 & (1.14) & 228 & $(0.80)$ & $1.39\left(0.026^{\#}\right)$ & 41 & $(0.98)$ & 184 & $(0.67)$ & $1.46\left(0.023^{\#}\right)$ \\
\hline Total patients & \multicolumn{2}{|c|}{$4,228(100.00)$} & \multicolumn{2}{|c|}{$28,521(100.00)$} & 32,749 & \multicolumn{2}{|c|}{$4,169(100.00)$} & \multicolumn{2}{|c|}{$27,639(100.00)$} & 31,808 \\
\hline
\end{tabular}

CCBs, calcium channel blockers; ACEIs, angiotensin converting enzyme inhibitors; ARBs, angiotensin II receptor blockers. *Percentage is calculated from total patients either with or without DM. Each patient might receive multiple drug classes. ${ }^{\#} p<0.05$ when compared the prevalence of patients with DM to patients without DM. 
Table 7. Anti-hypertensive drug prescription in patients with and without chronic kidney disease (CKD).

\begin{tabular}{|c|c|c|c|c|c|c|}
\hline \multirow[t]{2}{*}{$\begin{array}{l}\text { Anti-hypertensive } \\
\text { drug classes }\end{array}$} & \multicolumn{3}{|c|}{$\begin{array}{c}\mathbf{2 0 1 4} \\
\text { number }(\% *)\end{array}$} & \multicolumn{3}{|c|}{$\begin{array}{c}\mathbf{2 0 1 5} \\
\text { number }(\% *)\end{array}$} \\
\hline & With CKD & Without CKD & $\begin{array}{c}\text { Prevalence ratio } \\
(p \text {-value })\end{array}$ & With CKD & Without CKD & $\begin{array}{c}\text { Prevalence ratio } \\
(p \text {-value })\end{array}$ \\
\hline CCBs & $\begin{array}{ll}3,539 & (62.53)\end{array}$ & $14,835 \quad(54.76)$ & $1.14\left(<0.001^{\#}\right)$ & $2,985 \quad(62.58)$ & $15,366 \quad(56.83)$ & $1.10\left(<0.001^{\#}\right)$ \\
\hline ACEIs & $2,223 \quad(39.28)$ & 11,760 & $0.90\left(<0.001^{\#}\right)$ & $1,795 \quad(37.63)$ & 11,249 (41.60) & $0.90\left(<0.001^{\#}\right)$ \\
\hline Diuretic & $1,868 \quad(33.00)$ & $9,128 \quad(33.70)$ & $0.98 \quad(0.314)$ & $1,351 \quad(28.32)$ & $7,609 \quad(28.14)$ & $1.01(0.798)$ \\
\hline Anti-a & $1,913 \quad(33.80)$ & $8,133 \quad(30.02)$ & $1.13\left(<0.001^{\#}\right)$ & $1,535 \quad(32.18)$ & $7,547 \quad(27.91)$ & $1.15\left(<0.001^{\#}\right)$ \\
\hline ARBs & 813 (14.36) & $4,038 \quad(14.91)$ & $0.96 \quad(0.4$ & 861 & $4,659 \quad(17.23)$ & $1.05(0.168)$ \\
\hline Vaso & $391 \quad(6.91)$ & $638 \quad(2.36)$ & $2.93\left(<0.001^{\#}\right)$ & $422 \quad(8.85)$ & $666 \quad(2.46)$ & $3.59\left(<0.001^{\#}\right)$ \\
\hline Central agonist & $104 \quad(1.84)$ & $172 \quad(0.63)$ & $2.89\left(<0.001^{\#}\right)$ & $88 \quad(1.84)$ & $137 \quad(0.51)$ & $3.60\left(<0.001^{\#}\right)$ \\
\hline Total patients & $5,660(100.00)$ & $27,089(100.00)$ & 32,749 & $4,770(100.00)$ & $27,038(100.00)$ & 31,808 \\
\hline
\end{tabular}

CCBs, calcium channel blockers; ACEIs, angiotensin converting enzyme inhibitors; ARBs, angiotensin II receptor blockers. *Percentage is calculated from total patients either with or without CKD. Each patient might receive multiple drug classes. ${ }^{\#} p<0.05$ when compared the prevalence of patients with CKD to patients without CKD.

Table 8. Anti-hypertensive drug prescription in patients with and without left ventricular hypertrophy (LVH).

\begin{tabular}{|c|c|c|c|c|c|c|c|c|c|}
\hline \multirow[t]{2}{*}{$\begin{array}{l}\text { Anti-hypertensive } \\
\text { drug classes }\end{array}$} & \multicolumn{4}{|c|}{$\begin{array}{c}\mathbf{2 0 1 4} \\
\text { number }(\% *)\end{array}$} & \multicolumn{5}{|c|}{$\begin{array}{c}\mathbf{2 0 1 5} \\
\text { number }(\% *)\end{array}$} \\
\hline & With LVH & Withou & it $\mathrm{LVH}$ & $\begin{array}{c}\text { Prevalence ratio } \\
(p \text {-value })\end{array}$ & Wit & $\mathrm{iLVH}$ & Without & $\mathrm{t} \mathrm{LVH}$ & $\begin{array}{c}\text { Prevalence ratio } \\
(p \text {-value })\end{array}$ \\
\hline CCBs & $84(50.60)$ & 18,290 & $(56.13)$ & $0.90 \quad(0.152)$ & 59 & (55.66) & 18,292 & $(57.70)$ & $0.96(0.671)$ \\
\hline ACEIs & $68(40.96)$ & 13,915 & $(42.71)$ & $0.96 \quad(0.651)$ & 36 & $(33.96)$ & 13,008 & (41.30) & $0.82(0.140)$ \\
\hline Diuretics & $65(39.16)$ & 10,931 & $(33.55)$ & $1.17 \quad(0.127)$ & 39 & $(36.79)$ & 9,043 & $(28.53)$ & $1.29(0.060)$ \\
\hline Anti-adrenergics & $74(44.58)$ & 9,972 & $(30.60)$ & $1.46\left(<0.001^{\#}\right)$ & 35 & (33.02) & 8,925 & $(28.15)$ & $1.17(0.266)$ \\
\hline ARBs & 36 (21.69) & 4,815 & (14.78) & $1.47\left(0.012^{\#}\right)$ & 25 & (23.58) & 5,495 & (17.33) & $1.36(0.090)$ \\
\hline Vasodilators & $14 \quad(8.43)$ & 1,015 & $(3.12)$ & $2.71\left(<0.001^{\#}\right)$ & 4 & $(3.77)$ & 1,084 & $(3.42)$ & $1.10(0.841)$ \\
\hline Central agonists & $3 \quad(1.81)$ & 273 & $(0.84)$ & $2.16(0.173)$ & 2 & $(1.89)$ & 223 & $(0.70)$ & $2.70(0.147)$ \\
\hline Total patients & $166(100.00)$ & 32,583 & $(\mathbf{1 0 0 . 0 0 )}$ & 32,749 & 106 & $100.00)$ & $31,702($ & $(100.00)$ & $\mathbf{3 1 , 8 0 8}$ \\
\hline
\end{tabular}

CCBs, calcium channel blockers; ACEIs, angiotensin converting enzyme inhibitors; ARBs, angiotensin II receptor blockers. *Percentage is calculated from total patients either with or without LVH. Each patient might receive multiple drug classes. ${ }^{\#} p<0.05$ when compared the prevalence of patients with LVH to patients without $\mathrm{LVH}$.

Table 9. Correlation analysis between the number of anti-hypertensive drugs that a patient received and various variables.

\begin{tabular}{lcc}
\hline Variables & \multicolumn{2}{c}{ Spearman correlation coefficient $(\boldsymbol{p}$-value, $\boldsymbol{n})$} \\
\cline { 2 - 3 } & $\mathbf{2 0 1 4}$ & $\mathbf{2 0 1 5}$ \\
\hline Age & $0.027\left(<0.001^{*}, 32,688\right)$ & $0.023\left(<0.001^{*}, 31,806\right)$ \\
Waist circumference & $0.110\left(<0.001^{*}, 22,074\right)$ & $0.115\left(<0.001^{*}, 23,235\right)$ \\
Systolic blood pressure & $0.122\left(<0.001^{*}, 32,749\right)$ & $0.132\left(<0.001^{*}, 31,808\right)$ \\
Diastolic blood pressure & $0.012\left(<0.030^{*}, 32,749\right)$ & $0.032\left(<0.001^{*}, 31,808\right)$ \\
\hline
\end{tabular}

Missing data: Age=61 (0.19\%) and $2(0.01 \%)$; Waist circumferences=10,675 (32.60\%) and 8,573 (26.95\%), in 2014 and 2015 , respectively.

significant, the correlation coefficients were relatively weak.

\section{DISCUSSION}

Demographic data indicated that the characteristics of patients in 2014 and 2015 were comparable. However, an obviously distinctive information between two years was the number of patients with metabolic syndrome, a cluster of multiple clinical findings, including elevated blood pressure, which has been recognized since $1998^{7}$. Unfortunately, the recording of metabolic syndrome in DMHT-DAMUS was originated in 2015. Despite the change of disease recognition, we did not observe a remarkable influence which could alter essential information between these years of the study.
Another point of interest was the sex that more than $60 \%$ of patients in our study was women. However, epidemiological data in 2014 revealed that overall prevalence of hypertension in Thailand was $25 \%$, and the prevalence tended to be slightly higher in men when compared to women $(26 \% \text { vs } 24 \%)^{8}$.

As a mono-therapy or in combinations with other drugs, CCBs were prescribed at the highest percentage. This popularity of CCBs is in line with a previous study which enrolled hypertensive out-patient in Thailand during $2007^{9}$. Until now, the only absolute contraindication of CCBs, in particular non-dihydropyridine group, is chronic congestive heart failure. However, the use of newer dihydropyridine CCBs have been considered to be neutral in this type of cardiac abnormality ${ }^{10}$. A reason why CCBs were widely prescribed might be 
due to their relatively less requirement for general laboratory tests to examine hindered underlying factors, such as, severe renal impairment, hyperkalemia, bilateral renal artery stenosis, and pregnancy, as needed when prescribing ACEIs or ARBs ${ }^{6}$. In addition, this finding might be due to the patients in this database were relatively elderly, and CCBs appeared to be preferred in these vulnerable individuals.

According to Thai guidelines on the treatment of hypertension by Thai Hypertension Society in 2012, the latest national recommendation prior to our study period $^{6}$, hypertensive patients without co-morbid diseases who were younger than 55 years old could be initially treated by ACEIs or ARBs. This suggestion was due to the relative higher efficacy in controlling blood pressure, especially in non-elderly patients ${ }^{11}$. Nevertheless, we found that less than half of these young non-concurrent illness patients received ACEIs or ARBs as recommended. Again, this finding may imply that other factors, including ease and safe issue of initiating CCBs as previously discussed, could prevail the suggestion. Furthermore, despite less common for ARBs, the decision to initiate ACEIs or ARBs may be deny due to common adverse effects; for example, cough, angioedema, and drug hypersensitivity ${ }^{12}$.

The combination between CCBs and ACEIs was prescribed in the highest percentage among hypertensive patients without co-morbid diseases. This finding was in line with the recommendation in the guideline

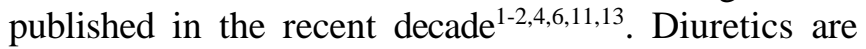
another class of anti-hypertensive drug suggested to be combined because of their distinctive pharmacological actions beyond CCBs and ACEIs/ARBs; nevertheless, metabolic adverse effects and electrolyte imbalances limit their usage 6 . Still, despite the concern on metabolic changes including hyperglycemia, considerable hypertensive patients with diabetes received diuretics. This finding may possibly due to the onset of diabetes might occur by other associated causes after patients had taken diuretics $^{14}$ or the lack of caution on patient's background in drug selection. Interestingly, we also found the prescription between ACEIs and ARBs, although the prevalence was relatively low $(0.23 \%$ in 2014 and $0.18 \%$ in 2015). However, this problematic prescription could be revised because the patients might carry higher harmful risks than beneficial effects ${ }^{15}$.

Interestingly, we found that anti-adrenergics, vasodilators, and central agonists were prescribed as a mono-therapy. Despite a lack of recommendation, it would be possible that these drugs were given to hypertensive patients with specific co-morbid diseases; for example, alpha-blockers for benign prostrate hyperplasia, beta-blockers for coronary artery disease, and central agonists (methyldopa) for pregnancy ${ }^{6}$. Unfortunately, the number of patients with these conditions were inadequate for the analysis. Thus, the acquisition of these co-morbid diseases in the database should be systematically improved as did for DM and CKD. It is worthwhile to mention that $0.11 \%$ and $0.12 \%$ of total patients in 2014 and 2015 received PAIs in combination with other anti-hypertensive drugs, whereas none of the patients received PAIs alone. This finding implied that the PAIs remained to be used as an add-on therapy. However, due to the number of patients who received PAIs being extremely small, we did not analyze their use in co-morbid diseases.

ACEIs, or alternatively ARBs, are recommended as the first choice in hypertensive patients with $\mathrm{DM}$ or CKD due to these agents can ameliorate diabetic nephropathy ${ }^{5-6}$ and albuminuria ${ }^{16}$. In line with the recommendation in patients with $\mathrm{DM}^{6,11}$, we found that the number of prescription of ACEIs plus the prescription of ARBs was $68.59 \%(49.36 \%+19.23 \%)$ in 2014 and $66.19 \%(42.84 \%+23.35 \%)$ in 2015 . In contrast to CKD patients, $62.53 \%$ and $62.58 \%$ received CCBs in 2014 and 2015, respectively. The popularity of CCBs might possibly relate with the concern on the use of ACEIs or ARBs in patients with severe renal impairment. Moreover, certain CCBs, such as manidipine and lercarnidipine, were proved to be beneficial for the reduction of albuminuria ${ }^{17-18}$. However, one may argue that ACEIs or ARBs could be superior to CCBs for the amelioration of albuminuria in nephropathy of hypertensive patients ${ }^{19}$. Another finding was the prevalence of hypertensive patients with LVH who received ACEIs, the first-line recommendation ${ }^{6}$, was less than that of non-LVH patients. However, these patients might receive ARBs, beta-blockers, diuretics, or even CCBs, which could be beneficial in the management of $\mathrm{LVH}$ instead $^{1,4}$

Herewith, the correlation between the number of anti-hypertensive drugs that a patient received and age, waist circumference, and systolic/diastolic blood pressure were also evaluated. Although all of the tests showed positive correlation with statistically significant, the degree of each association is relatively low. Thus, the statistical significance would be due to the vast of sample size. Of note, besides anti-hypertensive medicines, we tested the correlation among other variables and found the negative correlation between age of patients and DBP, as well as positive correlation between SBP and DBP in both years.

A strength of our study was the huge number of samples size and multi-center data retrieved from all regions of Thailand. Nevertheless, the anti-hypertensive drug for individual which was recorded as classes could be the most obvious limitation. Consequently, we were unable to enumerate the prevalence of particular drug use among various classes. In addition, all information in the database were derived from patients in Ministry of Public Health hospitals, of which the payment for majority of patients was covered by the universal cove- 
rage scheme. Therefore, the role of anti-hypertensive medicines which were not consisted in the Thailand National List of Essential Medicines (NLEM) might be trivial. Since this big database had no details of prescribers and disease severity, the prevalence of drug prescription in our study might not be assessed as the prescribing pattern in clinical practice. Lastly, despite currently unavailable, the analysis on an updated DMHT-DAMUS database could be beneficial. Nonetheless, the information gain from this study should partly contribute to the recent knowledge of anti-hypertensive drug prescription in Thai patients. In addition, possible inappropriate prescriptions which were revealed in our study could be a piece of evidence for the refinement of rational drug use in Thailand.

\section{CONCLUSIONS}

CCBs were the most prescribed anti-hypertensive medicine, both used as a mono-therapy or in combination with other medicines, irrespective of co-morbid diseases or age, although ACEIs, or alternatively ARBs, are recommended as the first-line agent for the management of hypertension in multiple concurrent conditions especially in patients with DM, CKD, and LVH. However, it should be noticed that the high utilization of CCBs might reflect that the patients in this study were elderly. Results from our study implicate that the pattern of anti-hypertensive medicine prescription in Thailand was partially in line with the recommendations in clinical practice guideline at that period. In addition, certain potential problematic prescriptions were found in a minor part of patients.

\section{ACKNOWLEDGEMENT}

We would like to thank Thailand National Health Security Office and MedResNet for kindly providing the information from the database.

\section{Conflict of interest:}

No conflict of interest.

\section{Funding}

None to declare.

\section{Ethics approval}

None to declare.

\author{
Article info: \\ Received May 23, 2021 \\ Received in revised form September 12, 2021 \\ Accepted October 2, 2021
}

\section{REFERENCES}

1. Verdecchia P, Reboldi G, Angeli F. The 2020 International
Society of Hypertension global hypertension practice guidelineskey messages and clinical considerations. Eur J Intern Med. 2020;82:1-6.

2. Thai Hypertension Society. Hypertension guideline 2019 [document on the Internet]. [cited 2021 May 21]. Available from: http://thaihypertension.org/files/HT\%20guideline\%202019.wit $\mathrm{h} \% 20$ watermark.pdf.

3. Ettehad D, Emdin CA, Kiran A, Anderson SG, Callender T, Emberson J, et al. Blood pressure lowering for prevention of cardiovascular disease and death: a systematic review and metaanalysis. Lancet Lond Engl. 2016;387(10022):957-67.

4. James PA, Oparil S, Carter BL, Cushman WC, Dennison-Himmelfarb C, Handler J, et al. 2014 Evidence-based guideline for the management of high blood pressure in adults: report from the panel members appointed to the eighth Joint National Committee (JNC 8). JAMA. 2014;311(5):507-20.

5. Van Buren PN, Toto R. Hypertension in diabetic nephropathy: epidemiology, mechanisms, and management. Adv Chronic Kidney Dis. 2011;18(1):28-41.

6. Thai Hypertension Society. Hypertension guideline 2012 [document on the Internet]. [cited 2021 May 10]. Available from: http://www.thaihypertension.org/files/216_1.Hypertension_Gui deline_2012.pdf.

7. Oda E. Historical perspectives of the metabolic syndrome. Clin Dermatol. 2018;36(1):3-8.

8. Health Systems Research Institute. The fifth national health exam 2015 [document on the Internet]. [cited 2021 May 10]. Available from: https://www.hsri.or.th/researcher/research/newrelease/detail/7711.

9. Buranakitjaroen P. Hypertension audit in clinical practice based in Thailand (HABIT). J Med Assoc Thai. 2011;94(Suppl 1):S57-65.

10. de Vries RJ, van Veldhuisen DJ, Dunselman PH. Efficacy and safety of calcium channel blockers in heart failure: focus on recent trials with second-generation dihydropyridines. Am Heart J. 2000;139(2 Pt 1):185-94.

11. McManus RJ, Caulfield M, Williams B, National Institute for Health and Clinical Excellence. NICE hypertension guideline 2011: evidence based evolution. BMJ. 2012;344:e181.

12. Messerli FH, Bangalore S, Bavishi C, Rimoldi SF. Angiotensinconverting enzyme inhibitors in hypertension: to use or not to use?. J Am Coll Cardiol. 2018;71(13):1474-82.

13. Thai Hypertension Society. Hypertension guideline 2015 [document on the Internet]. [cited 2021 May 10]. Available from: http://www.thaihypertension.org/files/GL\%20HT\%202015.pdf.

14. Mancia G. Preventing new-onset diabetes in thiazide-treated patients. Lancet Diabetes Endocrinol. 2016;4(2):90-2.

15. Misra S, Stevermer JJ. ACE inhibitors and ARBs: one or the other--not both--for high-risk patients. J Fam Pract. 2009;58(1): 24-7.

16. Pavlou DI, Paschou SA, Anagnostis P, Spartalis M, Spartalis E, Vryonidou A, et al. Hypertension in patients with type 2 diabetes mellitus: targets and management. Maturitas. 2018;112:71-7.

17. Fogari R, Mugellini A, Zoppi A, Lazzari P, Destro M, Rinaldi A, et al. Effect of successful hypertension control by manidipine or lisinopril on albuminuria and left ventricular mass in diabetic hypertensive patients with microalbuminuria. Eur J Clin Pharmacol. 2005;61(7):483-90.

18. Dalla Vestra M, Pozza G, Mosca A, Grazioli V, Lapolla A, Fioretto $\mathrm{P}$, et al. Effect of lercanidipine compared with ramipril on albumin excretion rate in hypertensive type 2 diabetic patients with microalbuminuria: DIAL study (diabete, ipertensione, albuminuria, lercanidipina). Diabetes Nutr Metab. 2004;17(5): 259-66.

19. Cativo EH, Lopez PD, Cativo DP, Atlas SA, Rosendorff C. The effect of calcium channel blockers on moderate or severe albuminuria in diabetic, hypertensive patients. Am J Med. 2021; 134(1):104-113.e3. 